\title{
On the Number of Close-and-Equal Pairs of Bits in a String (with Implications on the Security of RSA's L.S.B) \\ (Extended Abstract)
}

\author{
Oded Goldreich \\ Laboratory for Computer Science \\ MIT,room NE43-836,Cambridge,MA 02139
}

\begin{abstract}
We consider the following problem: Let $s$ be a $n$-bit string with $m$ ones and $n-m$ zeros. Denote by $C E_{t}(s)$ the number of pairs, of equal bits which are within distance $t$ apart, in the string $s$. What is the minimum value of $C E_{t}(\cdot)$, when the minimum is taken over all $n$-bit strings which consists of $m$ ones and $n-m$ zeros?

We prove a (reasonably) tight lower bound for this combinatorial problem.

Implications, on the cryptographic security of the least significant bit of a message encrypted by the RSA scheme, follow. E.g. under the assumption that the RSA is unbreakable; there exist no probabilistic polynomial-time algorithm which guesses the least significant bit of a message (correctly) with probability at least $\mathbf{0 . 7 2 5}$, when given the encryption of the message using the RSA. This is the best result known concerning the security of RSA's least significant bit.
\end{abstract}

Keywords: Cryptography, Combinatorial Analysis, the RSA Scheme, Bit Security, Combinatorial Bounds, Bit-String Properties, Public Key Cryptosystems.

Supported by a Weizmann Postdoctoral Fellowship 


\section{Introduction}

This paper combines a combinatorial study with the application of its results to the analysis of a cryptological question. (The combinatorial problem is fully defined and solved in Sec. 2.)

\subsection{Cryptological Background}

The importance of the notion of "partial information" to cryptographic research has gained wide recognition through the pionecring works of Blum and Micali [BM] and Goldwasser and Micali [GM]. In this paper we consider a much more specific question: the cryptographical security of the least significant bit of a message encrypted by the RSA scheme (hereafter referred to as RSA's l.s.b) .

The RSA encryption scheme was presented by Rivest, Shamir and Adleman [RSA]. It is the best known implementation of the notion of a Public Key Cryptosystem, which was suggested by Diffie and Hellman [DH]. Encryption using the RSA is done by raizing the message to a known exponent, $e$, and reducing the result modulo a known composite number, $N$, the factorization ${ }^{\prime}$ of which is kept secret. The inverse of $e$ in the multiplicative group $Z_{\varphi(N)}^{*}$ is used for decryption and is kept secret. It is widely believed that the RSA is hard to break. This means that an adversary who does not know the secret $\left(e^{-1} \bmod \varphi(N)\right)$ will not be able to compute the message from its encryption (i.e. to invert the encryption function).

However, even under this unbreakability assumption; it might be the case that the RSA leaks some "valuable" partial information. I.e. it might be that given the ciphertext, one can compute some function of half of the bits of the plaintext. Proving that, under the unbreakability assumption, this is infeasible will make the RSA much more attractive. This seems to be a high tool. Research attempts are meanwhile focused at the feasibility of guessing correctly the least significant bit of the plaintext (i.e. RSA's l.s.b.) $)^{2}$.

By saying that $R S A$ 's l.s.b is p-secure we mean that guessing it correctly with probability at least $p$ is as hard as inverting the RSA. Consider an oracle that when given the encryption (using the RSA) of a message guesses the least significant bit of the message correctly with probability $p$. Such an oracle will be called a p-oracle for $R S A$ 's l.s.b. Clearly, the existence of a polynomial time algorithm that inverts the RSA using a p-oracle for RSA's l.s.b implies that RSA's l.s.b is p-secure.

It is believed that RSA's l.s.b is $\left(\frac{1}{2}+\epsilon\right)$-secure, for arbitrary small constant $\epsilon$. Proving this statement might be a major breakthrough on the way to proving that any "valuable" partial information about the message encrypted by the RSA is as hard to get as inverting the RSA. Progress towards this goal has been slow but consistant, in the recent years.

\footnotetext{
1 To be exact, $N$ is the produce of two large primes, $p$ and $q . \varphi(\cdot)$ is the Euler's totient function, thus $\varphi(p q)=(p-1)(q-1)$.

${ }^{2}$ Nevertheless, results have been achieved also w.r.t. other kinds of partial information. For details consult [BCS] and [VV2].
} 
The first step was taken by Goldwasser Micali and Tong [GMT] who proved that RSA's l.s.b is $\left(1-\frac{1}{|N|}\right)$-secure, where $|N|$ is the size of the RSA's modulus.

Ben-Or, Chor and Shamir greatly improved this result by proving that RSA's l.s.b is $\left(\frac{3}{4}+c\right)$-secure, where $\epsilon$ is fixed and arbitrary small. Their paper [BCS] contains an algorithm which inverts the RS $\Lambda$ function. Their algorithm uses a $\left(\frac{3}{4}+t\right)$-oracle for RSA's l.s.b (in order) to decermine the parities of certain multiples of the original message. For further details consult [BCS] or [VV2].

Vazirani and Vazirani [VV1] have presented a very sophisticated modification of the algorithmic procedure used by Ben-Or, Chor and Shamir. The theme of their modification is a much betier use of the oracle answers. They showed that their modification is guaranteed to succeed when given access to a 0.741 -oracle for RSA's l.s.b. Recently, they have improved their analysis by showing that their modification is guaranteed to succeed even if it uses a 0.732 -oracle.

Using the combinatorial results obtained in this paper, we show that the Vazirani and Vazirani algorithm is guaranteed to succeed when it uses a 0.725 -oracle for RSA's l.s.b. Other observations w.r.t the Vazirani and Vazirani algorithm as well as w.r.t other inverting algorithms are also implied.

\subsection{Our Results}

The following problem occured to us when trying to improve Ben-Or, Chor and Shamir's result [BCS]:

Let $s$ be a $n$-bit string with $m$ ones and $n-m$ zeros. Two bits in the string $s$ are said to be $t$-close if they are within distance $t$ apart. Denote by $C E_{t}(s)$ the number of pairs of equal $t$-close bits in the string $s$. What is the minimum value of $C E_{t}(\cdot)$, over all $n$-bit strings which consists of $m$ ones and $n-m$ zeros?

In Sec. 2 we prove a (reasonably) tight lower bound on this combinatorial problem. With respect to proving the "amount" of security of the least significant bit of the RSA, this is a double-edged-sword:

(1) It provides a powerful tool for analyzing certain algorithms for inverting the RSA using an $\left(\frac{1}{2}+\delta\right)$-oracle for RSA's l.s.b .

For example the algorithm proposed by Vazirani and Vazirani [VV1] is shown to work when it uses any 0.725 -oracle for RSA's l.s.b (i.e. $\delta=0.225$ ). This establishes the best result known conserning the security of RSA's l.s.b .

(2) It points out the weakness of various proof techniques for determining the cryptographic security of RSA's l.s.b .

For example the Vazirani and Vazirani algorithm [VV1] may fail to invert if it uses a $\frac{2}{3}$-oracle for RSA's l.s.b.

These implications will be discussed in Sec. 3 . We believe that the combinatorial result has also other implications. 


\section{The Combinatorial Results}

In this section we give a formal definition of the combinatorial problem, discussed in the introduction, and prove a (reasonably) tight lower bound on it.

\subsection{Definitjons}

Let $s=\left(s_{0}, s_{1}, s_{2}, \ldots, s_{|s|-1}\right)$ be a binary string of length $|s|$. We denote by $s h_{i}(s)$ the string which result from $s$ by the application of $i$ left cyclic shifts. I.e:

$$
s h_{i}(s)=\left(s_{i}, s_{i+1}, s_{i+2}, \ldots, s_{i+|s|-1}\right),
$$

where indices are considered modulo $|s|$.

Define the i-overlap of a string, $s$, to be the number of positions which agree in $s$ and $s h_{i}(s)$. The $i$-overlap of $s$ will be denoted by over $_{i}(s)$, i.e.

$$
\left.\operatorname{over}_{i}(s)=\operatorname{Hamming}_{(s} \equiv \operatorname{sh}_{\mathfrak{i}}(s)\right) \text {, }
$$

where $\equiv$ denotes the bit by bit equal operation and $H a m m i n g(s)$ denotes the number of ones in $s$. Note that $\operatorname{over}_{i}(s)=\left|\left\{j: 0 \leq j<|s| \wedge s_{j}=s_{j+i}\right\}\right|$.

Denote by AverOver $(s, t)$ the average over the $i$-overlaps of $s$ for $i \in\{1,2, . ., t\}$. I.e.

$$
\operatorname{AverOver}(s, t)=\frac{1}{t} \sum_{i=1}^{t} \operatorname{over}_{i}(s)
$$

We remind the reader that $C E_{t}(s)$ was used to denote the number of pairs, of equal bits which are within distance $t$ apart, in the string $s$. I.e.

$$
C E_{t}(s)=\left|\left\{(i, j): 0 \leq i<j<n \wedge s_{i}=s_{j} \wedge j-i \leq t\right\}\right|,
$$

$$
\text { where } n=|s| \text {. }
$$

Clearly, $C E_{t}(s)=\sum_{i=1}^{t}\left|\left\{j: 0 \leq j<n \wedge s_{j}=s_{j+i}\right\}\right|$. Thus,

$$
C E_{t}(s)=t \cdot \operatorname{AverOver}(s, t) \text {. }
$$

When evaluating $C E_{t}(s)$ consider "lines" which connect equal $t$-close bits in $s$ (i.e. positions that contain equal values and are less than $t$ bits apart in the string $s$ ). These lines are hereafter called overlines. Note that $C E_{t}(s)$ is nothing but the number of overlines in the string $s$.

Let $n$ and $m$ be integers such that $0.5 n \leq m<n$. Let $\delta=\frac{m-0.5 n}{n}$. We denote by $S_{n}^{\delta}$ the set of $n$-bit binary strings with $m=(0.5+\delta) n$ ones (and $n-m$ zeros).

Denote by $\operatorname{Aver}(n, \delta, t)$ the minimum value of AverOver $(\cdot, t)$ divided by $n$, when minimized over all strings in $S_{n}^{\delta}$. I.e.

$$
\operatorname{Aver}(n, \delta, t)=\min _{s \in S_{n}^{\delta}}\left\{\frac{1}{n} \cdot \operatorname{AverOver}(s, t)\right\} \text {. }
$$

It is straightforward to see that for every $s \in S_{n}^{\delta}$, AverOver $(s, n)=\left(0.5+2 \delta^{2}\right) n$.

In this section we study $A \operatorname{ver}(n, \delta, t)$ for arbitrary $t, t<n$. We obtain non-trivial results, as the surprising fact that $\operatorname{Aver}(n, 0, t)$ converges to $\sqrt{2}-1 \approx 0.414$, when $\frac{n}{t}$ and $t$ are large enough. 


\subsection{P'roposilions}

We will assume throughout this section that $t \leq \frac{1}{2}(n-2)$. We will analyze $\Lambda$ ver $(n, \delta, t)$ as follows: Grst we will show that the minimum of $C E_{f}(\cdot)$ is achicved by strings which belong to a restricted subset of $S_{n}^{\gamma}$; and next we will minimize $C E_{t}(\cdot)$ over this subset. This will establish a lower bound on $\operatorname{Aver}(n, \delta, l)$. The upper bound will be implied by the proof of the lower bound, since this proof specifies a string $s \in S_{n}^{\delta}$ for which $C E_{t}(s) \approx n t \cdot A \operatorname{ver}(n, \delta, t)$.

\subsubsection{Reduction into a restricted subset}

In this subsection we will show that when analysing $A v e r(n, \delta, t)$ it is enough to consider strings in $S_{u}^{h}$ which have the following property:

The string contains no "short 3-alternations substring". A short 9-alternations substring is a substring of the form $\sigma \tau^{+} \sigma^{+} \tau$ and length less than $t+2$, where $\sigma \neq \tau \in\{0,1\}$. (Here, and throughout this paper, $\sigma^{+}$denotes a non-cmpty string of $\sigma$ 's.)

Proposition 1: $\operatorname{over}_{i}(s)=\operatorname{over}_{i}\left(\operatorname{sh}_{j}(s)\right)$

Prop. 1 follows directly from the definitions which consider strings as if they were cycles. From this point on, we also take the liberty of doing so.

The proofs of the following propositions are omitted; they can be found in the full version of this paper ([G84]).

Proposition 2: Let $\sigma_{j} \in\{0,1\}$, for $1 \leq j \leq 2 t$. Let $\alpha$ be a binary string. Let $n_{\tau_{1} \tau_{2}}=C E_{t}\left(\sigma_{1} \sigma_{2} \cdots \sigma_{t} \tau_{1} \tau_{2} \sigma_{t+1} \sigma_{t+2} \cdots \sigma_{2 t} \alpha\right)$. Then $n_{10}-n_{01}=2\left(\sigma_{1}-\sigma_{2 t}\right)$.

Note that switching $\tau_{1}$ and $\tau_{2}$ in the string $\sigma_{1} \sigma_{2} \cdots \sigma_{t} \tau_{1} \tau_{2} \sigma_{t+1} \sigma_{t+2} \cdots \sigma_{2 t} \alpha$ results in the string $\sigma_{1} \sigma_{2} \cdots \sigma_{t} \tau_{2} \tau_{1} \sigma_{t+1} \sigma_{t+2} \cdots \sigma_{2 t} \alpha$. The latter string has more overlines (than the former one) only if $\sigma_{1}=\tau_{2} \neq \tau_{1}=\sigma_{2 n}$. Note that the latter string has less overlines if $\sigma_{1}=\tau_{1} \neq \tau_{2}=\sigma_{2 n}$.

Proposition 3: Let $\alpha$ be a binary string and let $x, y, z, u$ be integers such that $x+y \geq t$ but $y+z<t$. Then:

(i) $C E_{t}\left(\sigma \tau^{x} \sigma^{y} \tau^{z-1} \sigma \tau \alpha\right) \leq C E_{t}\left(\sigma \tau^{x} \sigma^{y} \tau^{z} \sigma \alpha\right)$.

(ii) $C E_{t}\left(\sigma \tau^{x} \sigma^{y} \tau^{z-1} \sigma \tau \sigma^{u-1} \tau^{t-u} \sigma \alpha\right)<C E_{t}\left(\sigma \tau^{x} \sigma^{y} \tau^{z} \sigma^{u} \tau^{t-u} \sigma \alpha\right)$.

(iii) $C E_{t}\left(\sigma \tau^{x} \sigma^{y} \sigma \tau^{z} \alpha\right) \leq C E_{t}\left(\sigma \tau^{x} \sigma^{y} \tau^{z} \sigma \alpha\right)$.

Proposition 4: Let $s \in S_{n}^{\delta}$ be a binary string such that $C E_{t}(s)=n \cdot t \cdot \operatorname{Aver}(n, \delta, t)$. (I.e. $s$ is a string with minimum number of overlines among all strings in $S_{n}^{\delta}$.) Then there exist a string, $s^{\prime} \in S_{n}^{\delta}$, such that :

(i) The string $s^{t}$ contains a substring of the form $10^{+} 1^{+} 0$ the length of which is at least $t+2 .^{3}$

(ii) $C E_{t}\left(s^{\prime}\right)<C E_{t}(s)+t^{2}$.

${ }^{3}$ We remind the reader that $\sigma^{+}$denotes a non-emply string of $\sigma$ s. 
Proposition 5: let $s^{\prime} \in S_{n}^{\phi}$ be a string, with minimum number of overlines, which satisfies Prop. 4 . Then with no loss of generality, the string $s^{\prime}$ contains no substring of the form $10^{+} 1^{+} 0$ the length of which is less than $t+2$. Furthermore, the string $s^{\prime}$ contains at most one substring of the form $01^{+} 0^{+} 1$ the length of which is less than $t+2$.

We remind the reader that $C E_{t}\left(s^{\prime}\right)<n t A$ ver $(n, \delta, t)+t^{2}$ and that $s^{\prime} \in S_{n}^{\delta}$.

Proposition 6: Let $s^{\prime} \in S_{n}^{h}$ be a string as in Prop. 5. Then there exist a string $s^{\prime \prime} \in S_{n}^{\delta}$ such that:

(i) The string $s^{\prime \prime}$ contains no substring of the form $10^{+} 1^{+} 0$ the length of which is less than $t+2$.

(ii) The string $s^{\prime \prime}$ contains no substring of the form $01^{+} 0^{+} 1$ the length of which is less than $t+2$.

(iii) $C E_{t}\left(s^{\prime \prime}\right)<C E_{t}\left(s^{\prime}\right)+t^{2}$.

We remind the reader that our objective is to given a good lower bound on $\operatorname{Aver}(n, \delta, t)=\min _{s \in S_{n}^{b}} \frac{1}{n t} C E_{t}(s)$. Note that we have restricted our attention to strings that donot have short 3-alternations substrings; i.e. substrings of the form $01^{+} 0^{+} 1$ or $10^{+} 1^{+} 0$ which have length less than $t+2$. This is sufficient since there exist such a string, namely $s^{\prime \prime}$, that has approximately the minimun number of overlines. I.e. $C E_{t}\left(s^{\prime \prime}\right)<n t$ Aver $(n, \delta, t)+2 t^{2}$. Formally we define $R_{n}^{\delta}$ to be the set of strings which belong to $S_{n}^{\delta}$ and do not have short 3-alternating substrings. Aver ${ }_{R}(n, \delta, t)$ will denote $\min _{\tau \in R_{n}^{\delta}} \frac{1}{n t} C E_{t}(r)$. Clearly,

Proposition 7: Aver $(n, \delta, t) \leq \operatorname{Aver}_{R}(n, \delta, t)<\operatorname{Aver}(n, \delta, t)+\frac{2 t}{n}$.

Let us define even a more restricted subset of $S_{n}^{\delta}$ : The set $M R_{n}^{\delta}$ is the subset of strings which belong to $R_{n}^{\delta}$ and do not have long homogenous substrings; i.e. substring of the form $\sigma^{t+1}$, where $\sigma \in\{0,1\}$. Also, Aver $M R(n, \delta, t)$ will denote $\min _{\mathbf{r} \in M R_{n}^{\delta}} \frac{1}{n t} C E_{t}(r)$. Let us first give a tight lower bound on $\operatorname{Aver}_{M R}(n, \delta, t)$ and only later prove that this bound is approximately also a bound for $\operatorname{Aver}_{R}(n, \delta, t)$.

\subsubsection{Lower bound for $\operatorname{Aver}_{M R}(n, \delta, t)$}

Recall that each of the strings in $M R_{n}^{\delta} \subseteq S_{n}^{\delta}$ has the following properties:

(i) The string contains no short 3-alternating substrings.

(ii) The string contains no long homogenous substrings.

We will relay on the above properties of the strings in $M R_{n}^{\delta}$ in order to bound $\operatorname{Aver}_{M R}(n, \delta, t)$. Given a string $\tau \in M R_{n}^{\delta}$ we will introduce an expression, for $C E_{t}(r)$, which depends only on the numbers of bits in each maximal substrings of consecutive equal bits. In other words, we will introduce a localized counting of $C E_{t}(r)$.

Definition: We say that $b$ is a block (an all- $\sigma$ block) of the string $r$ if it is a maximal substring of equal bits. I.e. $b=\sigma^{+}$and $\tau=\tau b \tau \alpha$, where $\tau \neq \sigma \sigma$ and $\alpha$ is an arbitrary string. 
Denotations: Let $q$ denote the number of all-zero [all-one] blocks in $r$. Beginning from an arbitrary position between an all-one block and an all-zero block and going cyclically from left to right; number the blocks of consecutive zeros [ones] by $0,1,2, \ldots,(q-1)$. Denote by $z_{i}$ the number of zeros in the $i$-th all-zcro-block and by $y_{i}$ the number of ones in the $i$-th all-one-block. I.e., $r=0^{z_{0}} 1^{y_{0}} 0^{2} 1^{y_{1}} 0^{z-1} 1^{y_{1}} \ldots 0^{z_{q-1}} 1^{y_{q}-1}$.

Proposition 8: Overlines occur (in $r$ ) only either within a block or between two consecutive blocks (of the same bit).

Remark: Note that Prop. 8 holds even if $r \in R_{n}^{\delta}$.

This suggests to evaluate the number of overlines (in $r$ ) by counting the "contribution" of each (homogeneous) block to it. This counting is herealter referred as the Block-Localized Counting $(B L C)$ and proceeds as follows:

Block-Localized Counting (with respect to a block of length $l$ in $r$ ):

(i) The number of overlines within the block, denoted $I_{l}$.

(ii) The number of overlines between bits of the blocks neighbouring this block

(i.e the first block on its left and the first block on its right), denoted $B_{l}$.

Note that $I_{l}$ and $B_{l}$ are easy to evaluate and can be used to express $C E_{t}(r)$. Namely,

Proposition 9:

(i) $C E_{t}(r)=\sum_{i=0}^{q-1}\left(\left(I_{y_{i}}+B_{y_{i}}\right)+\left(I_{z_{i}}+B_{z_{i}}\right)\right)$, where $r=0^{z_{0}} 1^{y_{0}} 0^{z_{1}} 1^{y_{1}} \ldots 0^{z_{q-1}} 1^{y_{y-1}}$.

(ii) For $l<t, I_{l}=\left(\begin{array}{l}l \\ 2\end{array}\right)$ and $B_{l}=\sum_{i=1}^{t-l} i$.

(iii) For $l=t, I_{l}=\left(\begin{array}{l}t \\ 2\end{array}\right)$ and $B_{l}=0$.

Remark: Note that for $l>t, I_{t}=\left(\begin{array}{l}t \\ 2\end{array}\right)+(l-t) t$ and $B_{t}=0$. (Note that for $k>0$, $C E_{t}\left(\sigma^{t+k}\right)=C E_{t}\left(\sigma^{t+k-1}\right)+t=C E_{t}\left(\sigma^{t}\right)+k t$.) However such substrings donot exist in a string which belongs to $M R_{n}^{\delta}$.

Evaluating $I_{l}+B_{l}$ we get

Proposition 10: The contribution (to the BLC) of one l-bit long block (in $r$ ) is:

$$
f(l)=l^{2}-(t+1) l+\frac{t^{2}+t}{2} \text {. }
$$

Note that the contribution of all the all-zero blocks to the number of overlines (in $r$ ) only depends on the way the zeros are partitioned among the all-zero blocks. (I.e. it is independent of the way the ones are partitioned among the all-one blocks.) This contribution amounts to:

$$
g\left(z_{0}, z_{1}, \ldots, z_{q-1}\right)=\sum_{i=0}^{q-1} f\left(z_{i}\right)
$$

$$
\text { where } r=0^{z_{0}} 1^{y_{0}} 0^{z_{1}} 1^{y_{1}} \ldots 0^{z_{q-1}} 1^{y_{q-1}} \text {. }
$$

Note that $g(\cdot, \cdot, \cdots, \cdot)$ is a quadratic form and therefore

Proposition 11: For fixed $q, t$ and $k$, the minimum value of the function $g\left(x_{0}, x_{1}, \ldots, x_{q-1}\right)$ subject to the constraint $k=\sum_{i=0}^{q-1} x_{i}$, is obtained at $x_{0}=x_{1}=$ $\cdots=x_{q-1}=\frac{k}{q}$. 
Thus, the minimum number of overines is achieved if all the all-zero-blocks [all-oneblocks] are of the same size. This yiclds

Proposition 12: Let $Q=\left\{q \in\right.$ Integers: $\left.\frac{m}{i} \leq q \leq n-m\right\}$. Then:

$$
n t \Lambda \operatorname{ver}_{M M R}(n, \delta, t) \geq \min _{q C Q}\left\{q \cdot\left(f\left(\frac{m}{q}\right)+f\left(\frac{n-m}{q}\right)\right)\right\} .
$$

We remind the reader that $m=(0.5+\delta) n$.

Elaborating the r.h.s. expression of Prop. 12 we get

Proposition 13: $\operatorname{Aver}_{M k}(n, \delta, t) \geq \min _{g \in Q}\left\{h_{n}^{\delta}(q)\right\}$, where

$$
h_{n}^{\delta}(q)=\frac{t+1}{n} \cdot q+\frac{\left(0.5+2 \delta^{2}\right) n}{t} \cdot \frac{1}{q}-\frac{t+1}{t} .
$$

Note that

Proposition 14: The minimum of the function $h_{n}^{\delta}(\cdot)$ is obtained at:

$$
q_{\min }=\sqrt{\frac{0.5+2 \delta^{2}}{t(t+1)}} \cdot n
$$

and the minimum value, $h_{n}^{\delta}\left(q_{m i n}\right)$, is:

$$
v_{t}^{\delta}=\sqrt{\left(2+8 \delta^{2}\right) \cdot \frac{t+1}{t}}-\frac{t+1}{t} .
$$

Thus, Aver $\operatorname{AR}_{R}(n, \delta, t) \geq v_{t}^{\delta}$. All that is left is to derive a lower bound for $\operatorname{Aver}_{R}(n, \delta, t)$.

\subsubsection{Lower bound for $\operatorname{Aver}_{R}(n, \delta, t)$ and $\operatorname{Aver}(n, \delta, t)$}

In this subsection we show that a string, $r_{0} \in R_{n}^{\delta}$, with minimum overlines can be transformed into a string $r_{0}^{\prime} \in M R_{n^{\prime}}^{\delta^{\prime}}$, such that $n^{\prime} \approx n, \delta^{\prime} \approx \delta$ and $\left.C E_{(} r_{0}^{\prime}\right) \approx C E_{t}\left(r_{0}\right)$. We conclude by using this fact and the lower bound for $\operatorname{Aver}_{M R}(n, \delta, t)$, to introduce a lower bound for $\operatorname{Aver}_{R}(n, \delta, t)$.

Proposition 15: Let $r_{0} \in R_{n}^{\delta}$ be a string with minimum number of overlines; i.e. $C E_{t}\left(r_{0}\right)=n t$ Aver $_{R}(n, \delta, t)$. Then:

(i) For $\sigma \in\{0,1\}$, either $r_{0}$ contains no substring of more than $t$ consecutive $\sigma$ 's or $\tau_{0}$ contains no block of less than $t$ consecutive $\sigma$ 's. Futhermore, w.l.o.g, $r_{0}$ contains atmost one substring of more than $t$ consecutive $\sigma$ 's.

(ii) If $t>\frac{\frac{1}{2}+\delta}{\frac{1}{2}-\delta}$ then $r_{0}$ has no substring of the form $\sigma^{2 t}$.

(iii) If $t \leq \frac{\frac{1}{2}+\delta}{\frac{1}{2}-\delta}$ then $\operatorname{Aver}(n, \delta, t)=2 \delta$.

(iv) If $t>\frac{1}{\frac{1}{3}+\delta}$ then there exist a $k<t$, a $\delta^{\prime} \geq \delta$ and a $r_{0}^{\prime} \in M R_{n+k}^{\delta^{\prime}}$ such that $C E_{t}\left(r_{0}\right) \geq C E_{t}\left(r_{0}^{\prime}\right)-k t$.

We conclude by using Prop. $15_{(i v)}$ and the lower bound for Aver $M R(n, \delta, t)$, to introduce lower bounds for $\operatorname{Aver}_{R}(n, \delta, t)$ and $\operatorname{Aver}(n, \delta, t)$. 
Proposition 16: If $t>\overbrace{\frac{1}{2}}^{+\frac{\delta}{\delta}}$ then

(i) There exist $0 \leq k<t$ and $\delta^{\prime} \geq \delta$ such that $\operatorname{Aver}_{R}(n, \delta, t)>\operatorname{Aver}_{M R}\left(n+k, \delta^{\prime}, t\right)-\frac{\bar{t}}{n}$.

(ii) $\operatorname{Aver}_{R}(n, \delta, t)>v_{t}^{n}-\frac{t}{n}$.

(iii) $\operatorname{Aver}(n, \delta, t)>v_{t}^{\delta}-\frac{3 t}{n}$.

\subsection{The Main Results}

Throughout this section we assume that $\frac{\frac{1}{2}+\delta}{\frac{1}{2}-\delta}<t \leq \frac{1}{2}(n-2)$.

Lower Bound Lemma: Aver $(n, \delta, t)$ is $2^{t}$. least

$$
\left(\sqrt{\left(2+8 \delta^{2}\right) \cdot \frac{t+1}{t}}-\frac{t+1}{t}\right)-\frac{3 t}{n} .
$$

The proof follows immediately from Prop. 14 and $16_{(i i i)}$.

Upper Bound Lemma: Aver $(n, \delta, t)$ is at most

$$
\left(\sqrt{\left(2+8 \delta^{2}\right) \cdot \frac{t+1}{t}}-\frac{t+1}{t}\right)+\frac{t+1}{n}+\frac{1}{2 t^{2}} .
$$

The proof follows from observing that the proof of the lower bound specifies the structure of a string which achieves minimum $C E_{t}(\cdot)$ among all strings in $M R_{n}^{\delta}$. The only problem in constructing such a string is that non-integer numbers, of blocks and block sizes, may appear. However, the overlap added by the round-up of the number of blocks is less than $\frac{t+1}{n}$; while the overline added by the round-up of the blocks' sizes is less than $\frac{1}{2 t^{2}}$. For details see the full version of this paper.

Evaluating the expressions in the above lemmas we get

\section{Corollary 1:}

(i) $\sqrt{2}-1-O\left(\frac{1}{t}\right)<\operatorname{Aver}(n, 0, t)<\sqrt{2}-1+O\left(\frac{1}{t^{2}}\right)+O\left(\frac{t}{n}\right)$.

(ii) For $t \geq 2500$ and $n>300000 \cdot t$, Aver $(n, 0.177, t)>\frac{1}{2}+0.0001$.

(iii) For $t \geq 500$ and $n>10000 \cdot t, \operatorname{Aver}(n, 0.225, t)>0.55+0.0001$.

(iv) For every $2500<t<\frac{n}{10000}$ and $\delta \leq 0.176, \operatorname{Aver}(n, \delta, t)<\frac{1}{2}$.

(v) For every $500<t<\frac{n}{10000}$ and $\delta \leq 0.224, \operatorname{Aver}(n, \delta, t)<1-2 \delta$.

\subsection{Additional Definitions and Results}

In this section we define a different, yet related, combinatorial problem. Instead of considering the average overlap over all "small" ${ }^{4}$ shifts; we consider the maximum overlap obtained by one of the "small" shifts.

Let us define an $i$-overline to be a line which connects a pair of equal bits which are (exactly) at distance $i$ apart.

\footnotetext{
4Here, "small" means not greater than $t$.
} 
Denote by MaxOver $(s, t)$ the maximum over the $i$-overlaps of $s$ for $i \in\{1,2, \ldots, t\}$. J.e.

$$
\operatorname{Max} \operatorname{Over}(s, t)=\max _{1 \leq i \leq t}\left\{\operatorname{over}_{i}(s)\right\} .
$$

Denote by $\operatorname{Max}(n, \delta, t)$ the minimum value of $\operatorname{Max} O v e r(s, l)$ divided by $n$, when minimized over all strings in $S_{n}^{\delta}$. l.e.

$$
\operatorname{Max}(n, \delta, t)=\min _{s \in S_{n}^{b}}\left\{\frac{1}{n} \cdot \operatorname{Max} O \operatorname{ver}(s, t)\right\} .
$$

Clearly,

Proposition 17: $\operatorname{Max}(n, \delta, t) \geq \operatorname{Avcr}(n, \delta, t)$.

This establishes a trivial lower bound on $\operatorname{Max}(n, \delta, t)$. We donot beleive that this bound is tight; however we failed to prove a better one. On the other hand the following proposition yields an upper bound on $\operatorname{Max}(n, 0, t)$.

Proposition 18: ( $(\mathrm{i})$ is folklore and (ii) appears in van Lint[L])

(i) For every De-Bruijn Sequence ${ }^{5}, s$, of length $2^{k}$ and every $i, i \in\{1,2, \ldots, k-1\}$

$$
\operatorname{over}_{i}(s)=\frac{1}{2} \cdot 2^{k} \text {. }
$$

(ii) For every $k$ there exists a Shortened De-Bruijn Sequence ${ }^{6}, s$, of length $2^{k}-1$ such that for every $i, i \in\left\{1,2, \ldots, 2^{k}-2\right\}$,

$$
\operatorname{over}_{i}(s)=2^{k-1}-1 \approx \frac{1}{2} \cdot\left(2^{k}-1\right) .
$$

Using Prop. 18 we also obtain an upper bound on $\operatorname{Max}(n, \delta, t)$; i.e.

Proposition 19: [Here $q$ is an integer.]

(i) For $t+1=l=2^{k}-1, n=q l$ and $\delta=\frac{l+q-1}{2 n}, \operatorname{Max}(n, \delta, t) \leq \frac{1}{2}+\delta-\frac{1}{t+1}+\frac{1}{n}$.

(ii) $\operatorname{Max}(n, \delta, t) \leq \operatorname{Max}(n, \delta, t+1)$.

(iii) $\operatorname{Max}(n, \delta, t)<\frac{1}{2}+\delta+O\left(\frac{t}{n}\right)$.

The proof appears in the full version of this paper.

5 The $2^{k}$-bit long string $\left(s_{0}, s_{1}, s_{2}, \ldots, s_{2^{k}-1}\right)$ is a De-Bruijn Sequence if (when considered in circular order) it contain as substrings all possible bit-strings of length $k$.

${ }^{6}$ A Shortened De-Bruijn Sequence, of length $2^{k}-1$, is a $2^{k}$.long De-Bruijn Scquence in which a zero has been omitted from the all-zero block of length $k$. 


\section{On the Cryptographic Security of the RSA's L.S.B}

In this section we apply the results of the privious section to the analysis of algorithms which invert the RSA encryption function when given access to an oracle for the least significant bit of the encrypted message. This implies results (concerning the security of RSA.s I.s.b.) which fall into the following threc categories:

(i) A 0.725-security result (for RSA's l.s.b)

(ii) Conditional improvements of the above result. I.e. results which will hold if some conjecture is proven.

(iii) Bounds on the possibility of improvements using current techniques.

\subsection{Specific Background}

Our 0.725-security result is based on Vazirani and Vazirani work [VV1], which is an improvement of Ben-Or Chor and Shamir [BCS] work. In this subsection we sketch some of the ideas used in these nice works.

\subsubsection{A Sketch of Ben-Or Chor and Shamir Algorithmic Procedure}

\section{The essence of the Inverting Algorithm:}

The plaintext is reconstructed, from its encryption, by running a g.c.d procedure on two multiples ${ }^{2}$ of it. The values of these multiples (as well as the values of all multiples discussed hereafter) are "small" 8 . A Modified Binary G.C.D algorithm is used. To operate, this algorithm needs to know the parity of multiples of the plaintext. Thus, it is provided with a subroutine that determines the parity of these multiples.(see [BCS])

\section{Determining Parity using an Oracle which may err:}

The subroutine determines the parity of a multiple,$k x$, of the plaintext,$x$, by using an $\left(\frac{1}{2}+\delta\right)$-oracle for RSA's l.s.b as follows. It picks a random $r$ and asks the oracle for the parity (i.e. l.s.b) of both $r x$ and $r x+k x$ feeding it in turn with $E(r x)=E(r) E(x)$ and $E((r+k) x)=E(r+k) E(x)^{9}$. The oracle's answers are processed according to the following observation. Since $k x$ is "small" with very high probability $r x<r x+k x$. Then, the parity of $k x$ is equel to 0 if the parities of $r x$ and $r x+k x$ are identical; and equal to 1 otherwise. This is repeated many times; every repetition (instance) is called a $k x$-measurement (or a toss of the $k x$-coin). Note that the outcome of a $k x$-measurement is correct if the oracle was correct on both $r x$ and $r x+k x$. The outcome is correct also if the oracle was wrong on both queries (but this fact is not used in [BCS]).

\footnotetext{
7 All integers and operations are considered modulo, $N$, the RSA's modulus.

${ }^{8}$ Here and throughout the rest of the paper "small" means bounded by a very small fraction of the RSA's modulus.

${ }^{9} E(M)$ denokes the RSA encryption function. Recall that $E(M)=M^{e}(\bmod N)$, where $N$ and $e$ are respectively the IRSA's modulus and exponent.
} 
(Trivial) Measurement Analysis:

A $k x$-coin toss is correct with probability at least $2 \delta$.

(This suffices if $\delta=\frac{1}{4}+\epsilon$, see [BCS])

3.1.2. A Sketch of Vazirani and Vazirani Modification of the BCS-Procedure Distinguishing a Good Coin from a Bad one:

For $\delta<\frac{1}{1}$; if when running a Monte-Carlo experiment on a $k x$-coin toss, more than a $1-2 \delta$ fraction of the answers agree on some value, then this is the correct valuc.(In such a case the coin is said to be distinguishably good. See [VV1])

Using Distinguishably Good Coins:

Let $t$ be a fxed constant and $K$ be a set of cardinality $O(\log N)$. If for every $k \in K$ there exist a $1 \leq j \leq t$ such that the $(j \cdot k x)$-coin is distinguishably good then one can determine the parity of $k x$. (This is done by replacing every $k x$-measurement, of the subroutine, by a set of $O(\log \log N)$ measurements, see [VV1]). (The above condition will be referred to as the Distinguishability Condition.)

Vazirani and Vazirani combined the above sketched ideas to an algorithm that inverts the RSA using a $\left(\frac{1}{2}+\delta\right)$-oracle. It remained to be shown that when given certain or acles for RSA's l.s.b the Distinguishability Condition holds. In [VV1] Vazirani and Vazirani proved that the Distinguishability Condition holds for any 0.741-oracle for RSA's l.s.b.; in [VV2] they improved their analysis and showed that this condition holds for any 0.732 -oracle.

\subsection{Cryptographic Implications of our Combinatorial Results}

It is easy to show that the Distinguishability Condition is equivalent to the following condition, hereafter referred to as the Big-Advantage Condition : for some fixed $\mathrm{t}, \operatorname{Max}(N, \delta, t)>1-2 \delta+\epsilon$.

(Use oracle transformation through multiplication by the inverse of $k x \bmod N$. Note that if the inverse does not exist it is feasible to factor $N$ and inverting the RSA becomes easy.) This was also observed by Vazirani and Vazirani [VV2].

Thus, we can summerize Vazirani and Vazirani's [VV1] work by the following

VV-Theorem: Let $N$ be the RSA's modulus and $t$ be a fixed constant. If $\operatorname{Max}(N, \delta, t)>1-2 \delta+\epsilon$ then any $\left(\frac{1}{2}+\delta\right)$-oracle for RSA's l.s.b can be used to efficiently invert the RSA. (In other words: if the Big Advantage Condition holds for $\delta$ then RSA's l.s.b is $\left(\frac{1}{2}+\delta\right)$-secure.)

By our results, the Big-Advantage Condition holds for $\delta \geq 0.225$. Namely, using the VV-Theorem, Prop. 17 and Corollary $1_{(i i i)}$ we get

Corollary 2: Any 0.725-oracle for the least significant bit of the RSA can be efficiantly used to invert the RSA. 
In other words

Theorem: RSA's l.s.b. is 0.725 -secure.

Note that the result of corollary $1_{(i i)}$ is light. Thus under the condition Aver $(n, \delta, t)>1-2 \delta+c$, the result of Corollary 2 is optimal. However, $A \operatorname{ver}(n, \delta, t)>1-2 \delta+\epsilon$, is more than is needed to satisfy the Big-Advantage Condition. (Kecall that the Big-Advantage Condition requires only that $\operatorname{Max}(n, \delta, t)>1-2 \delta+\epsilon$.) Thus, any improvement of the current lower bound on $\operatorname{Max}(n, \delta, t)$ will yield an improvement of the result of Corollary 2. We beleive that $\operatorname{Max}(n, \delta, t)>A \operatorname{ver}(n, \delta, t)$ and thus that such an improvement is possible. Furthermore we conjecture that

Conjecture 1: $\operatorname{Max}(n, \delta, t) \approx \frac{1}{2}+\delta$.

Combined with the VV-Theorem this implies

Corollary 3: If Conjecture 1 is valid then RSA's 1.s.b. is $\left(\frac{2}{3}+\epsilon\right)$-secure, for arbitrary small fixed $\epsilon$.

Note that under the Big-Advantage Condition the "result" of Corollary 3 is optimal. This is due to Prop. $19_{(i i i)}$ which states that $\operatorname{Max}(n, \delta, t) \leq \frac{1}{2}+\delta$. Thus, using the VV-Theorem (or any proof technique which requires that the Big-Advantage Condition holds) one cannot hope to prove that RSA's l.s.b is $\frac{2}{3}$-secure.

Let us conclude by pointing out that the full power of the results obtained in section 2.3 was not used; however, we conjecture that it can be used. Namely,

Conjecture 2: Let $N$ be the RSA's modulus and $t<<N$. If $\operatorname{Aver}(N, \delta, t)>\frac{1}{2}+\epsilon$ then any $\left(\frac{1}{2}+\delta\right)$-oracle for RSA's l.s.b can be used to efficiently invert the RSA. (In other words: if Aver $(N, \delta, t)>\frac{1}{2}+\epsilon$ then RSA's l.s.b is $\left(\frac{1}{2}+\delta\right)$-secure.)

The condition of the statement of Conjecture 2 is hereafter referred to as the AverageAdvantage Condition. By Corollary $1_{(i i)}$, the Average-Advantage Condition is satisfied by $\delta=0.177$; thus

Corollary 4: If Conjecture 2 is valid then the RSA's l.s.b is 0.677 -secure.

Note that $\delta=0.177$ is the minimum for which the Average-Advantage Condition is satisfied. Thus no progress beyond the $\delta=0.177$ point can be made through the Average-Advantage Condition; i.e. when relying on it one cannot hope to prove that RSA's l.s.b is 0.676-secure.

Note that in Corollary 4 the missing part to reach the stated result is the algorithm that will use the analysis. (The analysis of the question which oracles satisfy the Avarage-Advantage Condition is complete!) However, in the case of the Big-Advantage Condition improved results can still be achieved (just) by improving the analysis of the combinatorial problem (see Corollary 3 ). 


\section{Conclusion}

We have solved a combinatorial problem and have shown how to use this solution to improve knowledge on the security of RSA's l.s.b. We have also pointed out possible directions for further improvement of our result. Improved results can be obtained by either conducting a better combinatorial analysis of $\operatorname{Max}(\cdot, \cdot, \cdot)$ or by suggesting an inverting algorithm based on the $\Lambda$ verage-Advantage Condition.

However such improvements will not suffice to show that RSA l.s.b. is $\frac{2}{3}$-secure. We believe that any improvement in the results concerning the security of RSA's l.s.b, beyond the $\frac{2}{3}$ point (which is still out of reach), must make use of additional properties of the RSA.

\section{Epilogue}

Meanwhile, Schnorr and Alexi [SA84] proved that RSA 1.s.b is $\left(\frac{1}{2}+\epsilon\right)$-secure, for every fixed $\epsilon$. Thus, the above coclusions are no longer of interest.

Schnorr and Alexi's proof is based on guessing the parity of $O(\log \log N)$ randomly selected positions and using these positions in all measurements of Ben-Or, Chor and Shamir's algorithmic procedure. Thus, the oracle is queried only about one end-point of each measurement and the analysis is w.r.t single positions rather than being w.r.t pairs of close positions.

Further improvement was achieved by Chor and Goldreich [CG84], who proved that RSA l.s.b is $\left(\frac{1}{2}+\frac{1}{\log ^{2} N}\right)$-secure, for every fixed $c$.

\section{Acknowledgements}

I am indebted to Tom Leighton for teaching me how to count (overlaps). I would like to thank Ron Rivest for guiding me with his insightful suggestions. I thank Vijay Vazirani for a private presentation of the .741 result and for his remarks. It is my pleasure to thank Michael Ben-Or, Benny Chor, Shafi Goldwasser, Hans Heller, Silvio Micali, Gary Miller and Avi Wigderson for very helpful discussions, useful ideas and consistent encouragement.

I am most thankful to Dassi Levi for her unique existence. 


\section{References}

[BCS] Ben-Or,M., Chor,B., and Shamir,A., "On the Cryplogrsphic Securily of Single RSA Bits", 15th ACM Symp. on Theory of Computation, April 1983, pp. 421-430

[BM] Blum,M., and Micali,S., "How to Generate Cryptographically Strong Sequences of Pseudo-Random Bits", to appear in the SIAM Jour. on Computing

[CG84] Chor,B., and Goldrcich,O., "RSA least signilicant bits are $\frac{1}{2}+\frac{1}{\text { poly }(\log N)}$ Secure, MIT/LCS/TM-260, May 1984

[DH] Diffe,W., and Hellman,M.E., "New Directions in Cryptography", IEEE Trans. on Inform. Theory, Vol. IT-22, No. 6, November 1976, pp. 644-654

[G84] Goldreich,O., "On the Number of Close-and-Equal Pairs of Bits in a String (with Implications on the Security of RSA's L.s.b.)", MIT/LCS/TM-256, March 1984

[GM] Goldwasser,S., and Micali,S., "Probabilistic Encryption", to appear in the JCSS special issue from the 14 th STOC

[GMT] Goldwasser,S., Micali,S., and Tong,P., "Why and How to Establish a Private Code on a Public Network", Proc. of the 29rd IEEE Symp. on Foundation of Computer Science, November 1982, pp. 134-144

[L] van Lint,J.H., Combinatorial Theory Seminar, Eindhoven University of Technology, Lecture Notes in Mathematics, SpringVerlag, 1974, pp. 90-91.

[RSA] Rivest,R.L., Shamir,A., and Adleman,L., "A Method for Obtaining Digital Signature and Public Key Cryptosystems", Comm. of the ACM,Vol.21, February 1978, pp. 120-126

[SA84] Schnorr,C.P. and Alexi,W., "RSA Bits are $0.5+\epsilon$ Secure", this proceedings [VV1] Vazirani,U.V., and Vazirani,V.V., "RSA's I.s.b is .741 Secure", presented in Crypto89, August 1983.

[VV2] Vazirani,U.V., and Vazirani,V.V., "RSA Bits are .732 Secure", preprint, November 1983. 